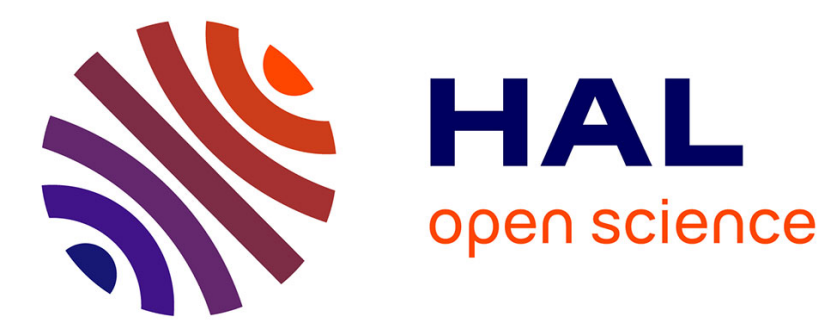

\title{
Intermolecular interaction in tetramethylurea solutions
}

L. Cser, T. Grósz, Yu. Ostanevich

\section{- To cite this version:}

L. Cser, T. Grósz, Yu. Ostanevich. Intermolecular interaction in tetramethylurea solutions. Journal de Physique IV Proceedings, 1993, 03 (C8), pp.C8-229-C8-232. 10.1051/.jp4:1993843 . jpa-00252274

\section{HAL Id: jpa-00252274 https://hal.science/jpa-00252274}

Submitted on 1 Jan 1993

HAL is a multi-disciplinary open access archive for the deposit and dissemination of scientific research documents, whether they are published or not. The documents may come from teaching and research institutions in France or abroad, or from public or private research centers.
L'archive ouverte pluridisciplinaire HAL, est destinée au dépôt et à la diffusion de documents scientifiques de niveau recherche, publiés ou non, émanant des établissements d'enseignement et de recherche français ou étrangers, des laboratoires publics ou privés. 


\section{Intermolecular interaction in tetramethylurea solutions}

\section{CSER, T. GRÓSZ and Yu.M. OSTANEVICH ${ }^{*}$}

Research Institute for Solid State Physics of the Hungarian Academy of Sciences, P.O. Box 49, 1525 Budapest, Hungary

* Joint Institute for Nuclear Research, Dubna 141980, Russia

Over its entire concentration range tetramethylurea (TMU) is miscible with water despite the fact that the TMU molecule is nonionic and contains four hydrophobic methyl groups.

Our previous small-angle neutron scattering (SANS) investigations revealed a number of interesting features of the structure of aqueous TMU solutions. By using a wide variety of the contrast variation method (both normal and deuterated TMU molecules were dissolved in heavy and light water) we were able to show that some structural changes take place in the aqueous TMU solution at an aquamolality of about 0.7 ; that the hydrophobic interaction between the TMU molecules becomes more attractive at higher temperature; that contact pairs are present in the relatively dilute solutions, that there is preferential orientation of water molecules in the hydration sphere [1].

It seems plausible that in the solution at least two types of interaction between the TMU molecules exist: a direct dipole-dipole and dispersion interaction between the solute molecules, and hydrophobic interaction which can lead to formation of solvent-separated and contact pairs. In addition to this two water molecules can form direct hydrogen bonds with the carbonyl group of TMU.

The present work aims to study these interactions in more details around the concentration of 0.7 aquamolality by carrying out a series of concentration dependent SANS measurements. A typical scattering pattern in Guinier coordinates is given in Fig 1. It is seen that the data can be well approximated by a straight-line, thus there are two parameters to be evaluated: the slope of the straight line and the scattered intensity at zero momentum transfer $(q=0), i(0)$.

The first parameter gives the so called apparent value of the radius of gyration $\left(R_{g, a p p}\right)$ while the second one, due to the use of a standard vanadium scatterer, can be transformed into the absolute scattering cross section of the molecule. These parameters are shown in Figs. 2 and 3. From these figures it can be seen that there is a very slight concentration dependence in the forward scattering cross-section and that the squared value of the apparent radius of gyration shows a nonlinear increase with increasing TMU concentration.

Such behaviour hints at the presence of attractive forces between the TMU molecules since a hard sphere repulsion force, which is of very short range, would give rise to a steep decrease in the forward scattering cross-section. On the other hand, the increase of the radius of gyration can be interpreted in terms of formation of aggregates. The simplest assumption would be that the aggregates can be represented by dimers; on the basis of this assumption, we perform a model calculation. The observed values of $i(0)$ and $R_{g, a p p}^{2}$ are averages which for two types of non-interacting particles can be written as

$$
i(0) \equiv d \sigma / d \Omega=i_{1}(0)+i_{2}(0)
$$


and

$$
\begin{gathered}
R_{g, a p p}^{2}=-(1 / 3) i^{-1}(0) d i / d q^{2} \equiv \\
R_{g, 1}^{2} i_{1}(0) /\left[i_{1}(0)+i_{2}(0)\right]+R_{g, 2}^{2} i_{2}(0) /\left[i_{1}(0)+i_{2}(0)\right]
\end{gathered}
$$

For interacting particles the whole effect of the interaction can be described by the coefficients appearing as integrals in the expression of the scattered intensity distribution. These integrals contain the pair correlation functions and the number of different interacting particles [2]

$$
\begin{aligned}
i(q)= & i_{0}(0) \bar{N}\left\{\sum p_{k}<F_{k}^{2}(q)>+\sum \sum p_{k} p_{j}<F_{k}(q)><F_{j}(q)>\left(1 / v_{1}\right) .\right. \\
& \left.\cdot \int_{0}^{\infty}\left[P_{k j}(r)-1\right](\sin (q r) / q r) 4 \pi r^{2} d r\right\}
\end{aligned}
$$

Where $F_{k}(q)$ designates the structure factor of the particle of type k; $p_{k}$, the probability that one of the $\bar{N}$ particles is of the type $\mathbf{k} ; v_{1}$, the average volume offered to each particle, regardless of its type; and $P_{k j}(r)$ gives in the first approximation the probability densities that the $\mathrm{k}$-th and $\mathrm{j}$-th type particles are separated by distance $r . \bar{N}$ is the average number of the particles in the irradiated volume $v$. The forward scattering intensity $i(q=0)$ for the system consisting of hard spheres can be easily calculated and the result of the calculation can be expressed as a linear function of the partial volumes $(\Phi)$ of the particles. For example for uniform spheres $i(0)=n[1-8 \Phi]$.

So far the interacting particles are concerned the coefficients of the partial volumes stemming from the integrals in eq. (3) at $q=0$ appear to be less simple, and for a mixture of aggregates one has to utilize some modelling approach. The integrals in eq. (3) then turn into coefficients $-B_{j k}$ - reflecting the interaction appearing between the $j$-th and $k$-th types of particle.

For simplicity, here we restricted ourselves to the consideration of pair correlation, in which case there are two main approaches to the model description of aggregate formation. According the first one we can consider the aggregates as relatively stable dimers in which two TMU monomers are bound via interaction the nature of which should be elucidated later. The theory of chemical equilibrium can be used for connecting the number densities of monomers $n_{m}$ and dimers $n_{d}$, in the equilibrium

$$
T M U+T M U \stackrel{K}{\rightleftharpoons}[T M U-T M U]
$$

Then

$$
n=n_{m}+2 n_{d} \quad \text { and } \quad K=n_{d} / n_{m}^{2}
$$

Here $n$ is the total number denstiy of the dissolved TMU molecules. Developing the idea discussed by Friedman [3] we deduced that $B_{m m}=8-2 K$. For $B_{d d}$ and $B_{m d}$ are expected to have values close to those of the hard sphere. (Indices $m$ and $d$ stand for monomers and dimers, respectively.) Combining expressions (1) and (3) the concentration dependence of the forward scattering intensity can be written as

$$
\begin{aligned}
i(0)= & i_{0}\left\{( n _ { m } / n ) \left[1-B_{m m} \Phi\left(n_{m} / n\right]+4\left(n_{d} / n\right)\left[1-B_{d d} \Phi\left(n_{d} / n\right)-\right.\right.\right. \\
& \left.-2\left(n_{m} / n\right)\left(n_{d} / n\right) B_{m d} \Phi\right\}
\end{aligned}
$$


In similar manner from eqs (2) and (3) the concentration dependence of the apparent value of the radius of gyration may be expressed as

$$
\begin{aligned}
R_{a p p}^{2} & =\left(n_{m} / n\right)\left[R_{g m}^{2}-B_{m m} \Phi\left(n_{m} / n\right)^{2}\left(R_{g m}^{2}+D_{m m}^{2}\right)\right]+ \\
& +4\left(n_{d} / n\right)\left[R_{g d}^{2}-B_{d d} \Phi\left(n_{d} / n\right)\left(R_{g d}^{2}+D_{d d}^{2}\right)\right]- \\
- & 2\left(n_{m} / n\right)\left(n_{d} / n\right) B_{m d} \Phi\left[(1 / 2)\left(R_{g m}^{2}+R_{g d}^{2}\right)+D_{m d}^{2}\right]
\end{aligned}
$$

where

$$
R_{g d}^{2}=R_{g m}^{2}+\frac{1}{4}(2 R+L)^{2}
$$

Here $R=3.6 \AA$ is the equivalent radius of the TMU molecules and $L$ is the distance between the TMU molecules. The interaction distances $\left(D_{i k}\right)$ were calculated in the hard sphere approximation by a method given elsewhere [1]. A least mean squares fit gives the set of parameters: $B_{m m}=8-(2 K=4.08+0.13) ; B_{d d}=-4.3+6 ; B_{m d}=7.3+5$; $L=6.2+1 \stackrel{\AA}{A}$;

From the value of $K$ it follows that a remarkable number of TMU molecules take part in dimer formation. In spite of the large statistical errors the low value of $B_{d d}$ indicates that the dimer-dimer interaction is rather attractive, while the coefficient $B_{m d}$ is within the limits of statistical error equal to 11.54 , corresponding to the hard sphere interaction. The relatively large value of $L$ tempts one to interpret this value as a distance between the oxygen atoms of two TMU molecules connected by intermediate water molecules via hydrogen bonds (see Fig. 4).

In the frame of the second approach one takes into consideration that in the dilute solutions the interactions between the solute molecules can be approximated by pair formation of two types: solvent separated and contact ones. In this case three different types of particle are present in the solution. In consequence, many more components appear in the pairwise interaction. It is evident that one has to decide which of these interactions are negligible. The answer to this question will be dealt with elsewhere. Despite giving relatively good approximation of the experimental data, even the first model suffers from an abundance of rough assumptions. However, the main advantage of the second approach is that its basic assumption allows us to connect observed data over wider range of concentration.

\section{References}

1. V.Yu.Bezzobotnov, L.Cser, T.Grósz, G.Jancsó, Yu.M.Ostanevich: Small-Angle Neutron Scattering in Aqueous Solutions of Tetramethylurea.

J.Phys.Chem. $\underline{96}, 976-982,(1992)$

2. A.Guinier, G.Fournet: Small-Angle Scattering of X-Rays, J.Wiley, New York (1955)

3. H.L.Friedman: A Course in Statistical Mechanics, Prentice-Hall, Englewood Cliffs, New Jersey (1985) 


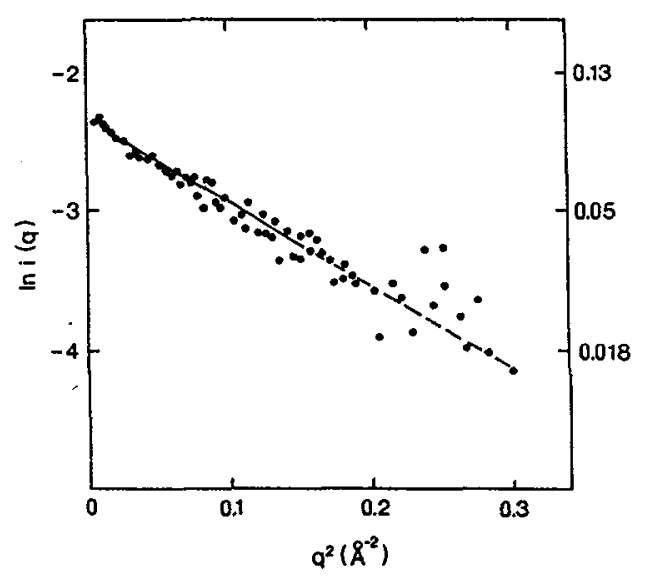

Figure 1: Guinier plot for a $1 \mathrm{~m}$ solution of $\mathrm{H}-$ TMU in $\mathrm{D}_{2} \mathrm{O}$, (i(q): scattering cross-section, $q$ - transferred momentum).

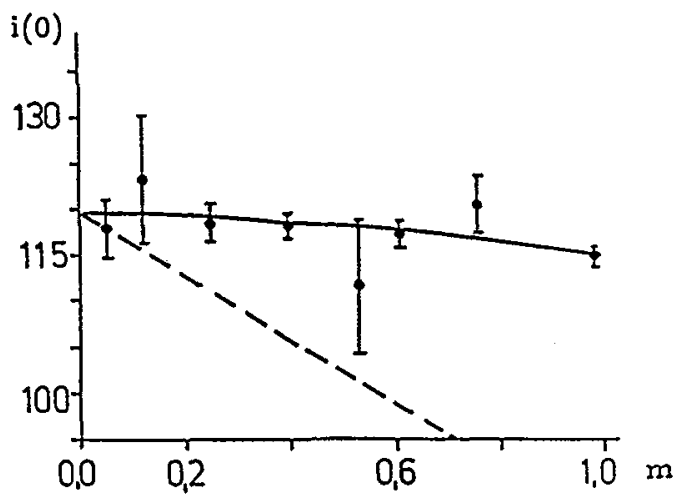

Figure 2: Forward scattering intensity crosssection of one TMU molecule (in barns) versus TMU concentration ( $m$, aquamolality). Full circles with error bars: experimental points, full line: result of least squares fit, broken line: hard-sphere calculation.

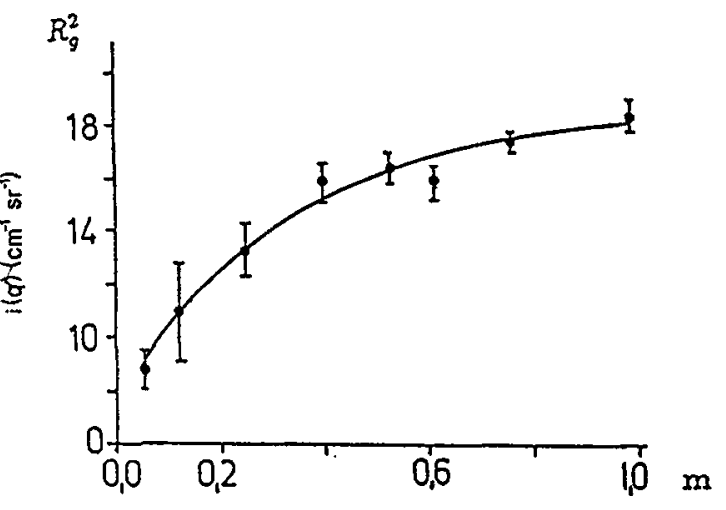

Figure 3: Squared value of radius of gyration (in $\AA^{2}$ ) versus concentration ( $m$, aquamolality) Full circles with error bars: experimental points; full line: result of leasts square fit.

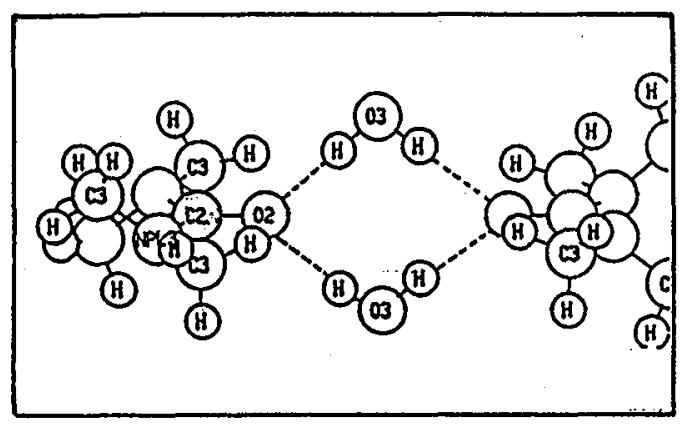

Figure 4: Possible view of hydrogen bonded dimer of the TMU molecules. 